\title{
High Resolution 3D Relativistic MHD Simulations of Jets
}

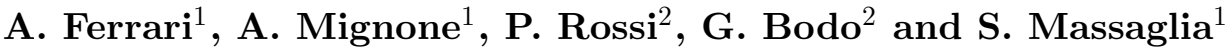 \\ ${ }^{1}$ Dipartimento di Fisica Generale dell Università di Torino, Italy, E-mail: ferrari@ph.unito.it \\ ${ }^{2}$ INAF Osservatorio Astronomico di Torino, Italy
}

\begin{abstract}
We performed high-resolution three dimensional numerical simulations of relativistic MHD jets carrying an initially toroidal magnetic field responsible for the process of jet acceleration and collimation. We find that in the $3 \mathrm{D}$ case the toroidal field gives rise to strong current driven kink instabilities leading to jet wiggling. However, it appears to be able to maintain an highly relativistic spine along its full length.
\end{abstract}

Keywords. Jets, numerical magneto-hydrodynamics, relativity, instabilities

In the most widely accepted models of relativistic jets from AGNs magnetic fields with footpoints attached to a spinning black hole or accretion disc are the necessary element for jet acceleration and collimation (e.g. see Narayan et al. 2007). In particular, it is likely that a toroidal component prevails at large scales as the poloidal component decays as $B_{p} \propto 1 / R_{j}^{2}$ while the toroidal $B_{\phi} \propto 1 / R_{j}$, where $R_{j}$ is the jet radius that increases from the inner galactic core to the radio jet lobes.

However, although jets must be Poynting-dominated at the origin, observational data suggest that they become kinetically dominated beyond about 1,000 gravitational radii from the central acceleration region, powered by a super-massive black-hole of $\sim 10^{8-10}$ $\mathrm{M}_{\odot}$ (Sikora et al. 2005).

Configurations in which toroidal magnetic fields dominate are known to be violently unstable to the $m=1$ kink instability according to the Kruskal-Shafranov criterion $\left|B_{\phi} / B_{p}\right|>2 \pi R_{j} / z$. The mechanism by which the toroidal field responsible for the jet acceleration can be dissipated may be related to the above mentioned instability (Giannios \& Spruit 2006). At the same time relativistic jets produced in proximity of rapidly spinning black-holes with dipolar magnetic fields expand rapidly and can possibly survive up to large radii without showing appreciable disruption (McKinney \& Blandford 2009).

Here we report on the first high-resolution 3D relativistic MHD simulations of the propagation of a magnetized jet injected in a uniform un-magnetized external medium. From the variety of possible magnetic field configurations, we consider the case of jets initially carrying a purely toroidal magnetic field, consistently with the previous discussion. $\mathrm{Nu}-$ merical simulations are carried out using the PLUTO code for astrophysical gasdynamics (Mignone et al. 2007). The chosen configuration employs a second order Godunov type scheme with the recently developed HLLD Riemann solver (Mignone et al. 2009) and the mixed hyperbolic/parabolic divergence cleaning to control the solenoidal constraint. The best overall resolution in our calculations corresponds to $640 \times 1600 \times 640$ computational zones with the radius of the beam spanning 20 zones.

The computational domain is initially filled with an un-magnetized medium at rest, with uniform density $\rho_{a}$ and gas pressure $p_{a}$. The jet flows is injected through a cylindrical nozzle with constant density $\rho_{j}$ and longitudinal velocity component given in terms of the Lorentz factor $\gamma_{j}$. 


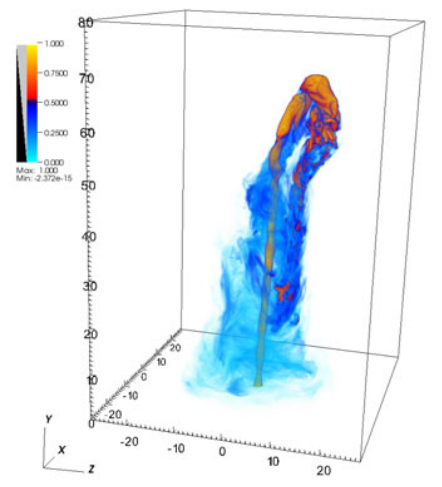

The typical structure of the jet when it has reached a length of 70 radii is given in the figure that shows the volume rendering of the tracer distribution.

The presence of a toroidal component of the field is known to drive current-driven kink instabilities which in our case are responsible for jet wiggling and beam deflection off the main longitudinal axis. The wandering of the jet head, induced by kink instability effects, may create multiple sites where the jet impacts on the external medium forming strong shocks. This behavior originates hot spots observed as in several radio jets. The asymmetry of the backflow is another feature that replicates the observational appearance of several objects.

An additional effect of the toroidal field component is a shielding of the inner jet core from any interaction with the surroundings and therefore from a loss of momentum. Correspondingly the jet remains highly relativistic all along its length. Quasi periodic shocks, formed by the combination of several effects like the interaction with the cocoon and the toroidal field pinching, can be observed all along the jet extension. A spine plus sheath layer structure with shocks is consistent with recent observations by the AGILE and FERMI missions and the TeV ground arrays (Donnarumma et al. 2009, Acciari et al. 2009) that jets at sub-kpc scale originate correlated variability at radio frequencies and in $\mathrm{X}$ and gamma rays.

\section{Acknowledgements}

This work was supported by research contracts with INAF and CINECA.

\section{References}

Acciari, V. A., Aliu, E., Arlen, T., et al. 2009, Science, 325, 444

Donnarumma, I., Vittorini, V., Vercellone, S., et al. 2008, ApJL, 691, L13

Giannios, D. \& Spruit, H. C., 2006, A\&A, 450, 887

McKinney, J. C. \& Blandford, R. D., 2009, MNRAS, 394, L126

Mignone, A., Bodo, G., Massaglia, S., Matsakos, T., Tesileanu, O., Zanni, C., \& Ferrari, A. 2007, ApJS, 170, 228

Mignone, A., Ugliano, M., \& Bodo, G. 2009, MNRAS, 393, 1141

Narayan, R., McKinney, J. C., \& Farmer, A.J. 2007, MNRAS, 375, 548

Sikora, M., Begelman, M. C., Madejski, G. M., \& Lasota, J.-P., 2005 ApJ, 625, 72 